\title{
Periodontitis is associated with a loss of colonization by Streptococcus sanguinis
}

\section{Correspondence \\ Catalina-Suzana Stingu \\ cstingu@iasi.mednet.ro}

Received 23 September 2007

Accepted 6 December 2007

\author{
Catalina-Suzana Stingu, ${ }^{1,2,3}$ † Klaus Eschrich, ${ }^{1}$ Arne C. Rodloff, ${ }^{2}$ \\ Reiner Schaumann ${ }^{2}$ and Holger Jentsch ${ }^{3}$
}

\author{
${ }^{1}$ Institute of Biochemistry, Medical Faculty, University of Leipzig, Johannisallee 30, 04103 Leipzig, \\ Germany \\ ${ }^{2}$ Institute for Medical Microbiology and Epidemiology of Infectious Diseases, University of Leipzig, \\ Liebigstr. 24, 04103 Leipzig, Germany \\ ${ }^{3}$ Department of Conservative Dentistry and Periodontology, University of Leipzig, Nürnberger \\ Straße 57, 04103 Leipzig, Germany
}

\begin{abstract}
The aim of this study was to estimate differences in the prevalence of oral streptococcal species in the subgingival biofilm of patients with aggressive periodontitis and of healthy controls.

Thirty-three patients with clinical and radiological proof of aggressive periodontitis and 20 healthy subjects were enrolled in this study. Clinical indices were recorded in a six-point measurement per tooth. Samples of the subgingival biofilm were taken with paper points from four teeth of each individual. Alpha- and non-haemolytic, small and catalase-negative colonies were biochemically identified using a rapid ID 32 STREP system and matrix-assisted laser desorption/ionization time-of-flight mass spectrometry. A total of 118 strains of oral streptococci (11 species) were identified and Streptococcus sanguinis was found significantly more often in healthy subjects $(P=0.001)$. Conversely, the absence of $S$. sanguinis was associated with high values of clinical indices $(P=0.001-0.002)$. Aggressive periodontitis seems to be associated with a loss of colonization of $S$. sanguinis. Whether or not $S$. sanguinis offers protection against aggressive periodontitis needs to be determined. Otherwise, there were no significant differences in the distribution of oral streptococcal species in patients and healthy subjects.
\end{abstract}

\section{INTRODUCTION}

Healthy gingivae are associated with a simple supragingival biofilm composition: a few (1-20) layers of oral streptococci, Gram-positive rods and very few Gram-negative cocci. These bacteria are early colonizers that are able to survive in an aerated environment. In contrast, clinical gingivitis is associated with the development of a more organized dental plaque of 100-300 layers, with anaerobic Gram-negative rods and filaments being predominant. The species involved in biofilm formation may vary depending on local environmental characteristics, but the colonization pattern is always the same (Marsh \& Bradshaw, 1999; Marsh, 2004).

Bacterial communities from dental biofilms tend to be grouped in clusters (complexes) according to nutritional and atmospheric requirements. The initiation and

†Present address: Department of Microbiology, Faculty of Dentistry, 'Gr. T. Popa' University of Medicine and Pharmacy, lasi, Romania.

Abbreviations: API, approximate plaque index; BOP, bleeding on probing; CAL, clinical attachment level; MALDI-TOF-MS, matrix-assisted laser desorption/ionization time-of-flight mass spectrometry; $\mathrm{OHI}$, oral hygiene index; PD, probing depth. progression of periodontitis is thought to be caused by several species belonging to 'red' and 'orange' complexes (Porphyromonas gingivalis, Tannerella forsythia and Treponema denticola, and Prevotella intermedia and Fusobacterium nucleatum, respectively) (Socransky et al., 1998). However, according to the 'ecological plaque hypothesis' (Marsh, 1991), the lack of so-called 'protective bacteria' is also thought to play an important role. These are microbial species that can occupy a niche that could shelter pathogenic organisms or that can inhibit some pathogens through metabolic antagonism or by directly inactivating them (Quirynen et al., 2001). Some of the members of the 'yellow' complex of oral streptococci are candidates for this position. Plaque samples from healthy gingival sulci normally contain a large number of oral streptococci (Theilade et al., 1966). Socransky et al. (1998) previously showed that yellow complexes, as a total, were associated with shallow pockets (probing depth of $<3 \mathrm{~mm}$ ). Thus colonization of certain oral streptococci might be one factor offering protection against periodontitis.

However, few data are available about the distribution of oral streptococci in subgingival biofilms of patients with aggressive periodontitis. Previous studies have focused on 
certain species of oral streptococci (Streptococcus sanguinis, Streptococcus mutans, Streptococcus intermedius and Streptococcus oralis) (Van der Reijden et al., 2001; Dowsett et al., 2002) or described the composition of subgingival biofilm in oral streptococci in healthy subjects (Frandsen et al., 1991), in diabetic patients with periodontitis (Hintao et al., 2007) or in patients with periodontitis.

The aim of this study was to estimate differences in the prevalence of oral streptococcal species in the subgingival biofilm of patients with aggressive periodontitis and of healthy controls.

\section{METHODS}

Patients and healthy control subjects. Thirty-three patients with aggressive periodontitis and 20 healthy subjects were enrolled in this prospective study. Diagnosis of aggressive periodontitis was made on clinical and radiographic findings, which showed rapid attachment loss and bone destruction (Wiebe \& Putnins, 2000). The patients included had at least 14 natural teeth, and at least four pockets (one in each quadrant) with a probing depth (PD) and clinical attachment level (CAL) of $\geqslant 4 \mathrm{~mm}$. Healthy controls had a PD of $\leqslant 3 \mathrm{~mm}$ and no attachment loss, and were matched with the patients for age, sex and smoking status. Patients were excluded from the study if they were pregnant, had other infectious diseases, had a history of previous periodontal treatment or if they had had antimicrobial therapy 6 months prior to the study. Demographic parameters and the history of smoking were obtained using a questionnaire. The Ethical Committee of the Faculty of Medicine, University of Leipzig, Germany, approved the protocol, including clinical measurements and the sampling procedure. All subjects were informed of the nature, potential risks and benefits of participation in the study, and signed informed consent prior to entry into the study.

Clinical measurements. Measurements of approximate plaque index (API; +/-), oral hygiene index (OHI; $0-3)$, gingival index (Lobene; $0-4)$, bleeding on probing (BOP; $+/-)$, PD and CAL were recorded in a six-point measurement per tooth (mesiobuccal, buccal, distobuccal, distolingual, lingual and mesiolingual) for all teeth. For clinical recordings of PD and CAL, a periodontal probe (Hu-Friedy) was used.

Microbiological assessment. Samples of the subgingival biofilm were taken with paper points (ISO 50) from four teeth (one per quadrant) of each individual. The teeth were chosen based on clinical measurements as the teeth with the highest value of PD and CAL, and samples were taken from the deepest site of the tooth. The supragingival plaque was removed and contamination with saliva was avoided. Two paper points were used for one site and were kept in situ for $10 \mathrm{~s}$ each. All paper points from one individual were then immersed in $1 \mathrm{ml}$ thioglycolate broth and immediately taken to the laboratory. Broth dilutions $\left(10^{-2}-10^{-5}\right)$ were cultured on sheep blood agar at $37{ }^{\circ} \mathrm{C}$ in an aerobic atmosphere for $48 \mathrm{~h}$. Alpha- or non-haemolytic, small and catalase-negative colonies were identified biochemically using a rapid ID 32 STREP system (bioMérieux). All identified Streptococcus isolates were also subjected to matrix-assisted laser desorption/ionization time-of-flight mass spectrometry (MALDI-TOF-MS) analysis.

MALDI-TOF-MS sample preparation. Individual colonies of streptococcal isolates were recultured in brain-heart infusion broth overnight and then prepared according to Friedrichs et al. (2007). The bacterial suspension $(1 \mathrm{ml}$ ) was centrifuged at $8500 \mathrm{~g}$ for $15 \mathrm{~min}$. The sediment was washed twice with DNase-free water (Sigma), dissolved in $50 \mu \mathrm{l} 80 \%$ trifluoroacetic acid (TFA; Merck) and left for $10 \mathrm{~min}$ at room temperature. DNase-free water $(150 \mu \mathrm{l})$ and $200 \mu \mathrm{l}$ acetonitrile (ACN; Sigma) were added. The samples were stored at $-20{ }^{\circ} \mathrm{C}$. After thawing, the samples were centrifuged at $13000 \mathrm{~g}$ for $2 \mathrm{~min}$. The supernatant was transferred into a $1.5 \mathrm{ml}$ Eppendorf tube and dried in a vacuum centrifuge. The pellet was dissolved in $20 \mu \mathrm{l} 2.5 \%$ TFA/ $50 \%$ ACN. One microlitre was pipetted onto a stainless-steel MALDI target plate. For each strain, five or ten consecutive spots were prepared. After drying, the spots were covered with $1 \mu \mathrm{l}$ matrix $(\alpha-$ cyano-4-hydroxycinnamic acid, saturated solution in 2.5\% TFA/50 \% ACN). The matrix/sample spots were crystallized by air drying. In order to demonstrate reproducibility, the sample preparation was repeated for each strain, starting with a new culture.

MALDI-TOF-MS analysis. The MALDI-TOF-MS procedures have been described in detail elsewhere (Friedrichs et al., 2007). Briefly, all mass spectra were acquired using an Autoflex II (Bruker Daltonics) MALDI-TOF mass spectrometer with a nitrogen laser $(337 \mathrm{~nm})$ operated in positive linear mode (delay $150 \mathrm{~ns}$, voltage $20 \mathrm{kV}$, mass range $2-50 \mathrm{kDa}$ ) using Flexcontrol software version 2.4 (Bruker Daltonics). Each spectrum was obtained by averaging 500 laser shots acquired in automatic mode at the minimum laser power necessary for ionization of the sample. The spectra were calibrated externally using a standard calibrant mixture (Protein Calibration Standard I; Bruker Daltonics). The data files were transferred to Flexanalysis version 2.4 (Bruker Daltonics) for automated peak extraction.

Using the software Flexanalysis, 40 peaks were automatically labelled in each spectrum according to their appearance above the background (threshold ratio 1.5). Correct labelling was controlled manually. Peak lists containing masses and intensities were exported as ASCII files. Similarity analysis between peak lists was carried out using a hierarchical clustering procedure performed with MatLab 7.3 (The MathWorks). The reproducibility of the method was shown by the similarity of spectra belonging to the same species. Similarity analysis with the peak lists obtained from spectra of the reference strains and some clinical isolates that were identified previously by sequence analysis of the 16S rRNA gene showed that all of the spectra belonging to one species clustered together. No outliers were observed. The database used for identification contained 20 species of oral streptococci identified by PCR and sequence analysis of the $16 \mathrm{~S}$ rRNA gene. For cases where Streptococcus mitis or S. oralis could not be identified unambiguously, we used a support vector machine algorithm. Support vector machines are a class of statistical learning algorithm whose theoretical basis was first presented by Vapnik (1995).

Data analysis. A univariate description was used to analyse all clinical and bacteriological data. To determine differences in the prevalence of oral streptococcal species in the subgingival biofilm of patients with aggressive periodontitis and of healthy controls, $\chi^{2}$ analysis and a Mann-Whitney U-test were used. To account for multiplicity, the alpha level was lowered from 0.05 to 0.003 using Bonferroni adjustment.

\section{RESULTS AND DISCUSSION}

The clinical data of the aggressive periodontitis patients and periodontally healthy subjects are summarized in Table 1 . The two groups of patients had comparable mean ages, the range being 20-69 years of age. Thirty-seven $(69.8 \%)$ were women and $20(37.7 \%)$ were smokers. The mean PD and attachment loss of sampled sites were higher than the mean for all teeth in all patients with aggressive periodontitis. 
Table 1. Mean clinical parameters of the subjects enrolled in this study

\begin{tabular}{|lcc|}
\hline Parameter & $\begin{array}{c}\text { Periodontitis patients } \\
(\text { mean } \pm \text { SD, } \boldsymbol{n}=\mathbf{3 3})\end{array}$ & $\begin{array}{c}\text { Healthy subjects } \\
(\text { mean } \pm \text { SD, } \boldsymbol{n}=\mathbf{2 0})\end{array}$ \\
\hline Age (years) & $39.39 \pm 10.47$ & $37.65 \pm 10.88$ \\
API (\%) & $41.35 \pm 30.58$ & $15.77 \pm 12.56$ \\
OHI (0-3) & $0.41 \pm 0.39$ & $0.06 \pm 0.07$ \\
Lobene (0-4) & $0.82 \pm 0.62$ & $0.05 \pm 0.03$ \\
BOP (\%) & $44.20 \pm 24.96$ & $3.09 \pm 3.32$ \\
CAL (total) & $3.73 \pm 0.85$ & - \\
CAL (sites) $\dagger$ & $4.79 \pm 1.28$ & - \\
PD (total) & $3.50 \pm 0.73$ & $1.69 \pm 0.28$ \\
PD (sites) $\dagger$ & $4.36 \pm 1.10$ & $1.59 \pm 0.24$ \\
& & \\
\hline
\end{tabular}

${ }^{*}$ Mean of all teeth.

$\dagger$ Mean of the four sampling sites.

From both groups, a total of 134 isolates were tested using the rapid ID 32 STREP system (bioMérieux). Strains that were not identified as belonging to the genus Streptococcus were not studied further, and the remaining 118 streptococcal isolates ( 73 isolates from patients, 45 isolates from control subjects) were subjected to MALDI-TOF-MS analysis. The distribution of species of oral streptococci in both patients and control subjects is shown in Table 2. Among the 11 streptococcal species isolated, S. oralis (38 isolates), S. sanguinis (33 isolates) and S. mitis (20 isolates) were the most prevalent. S. sanguinis was found in 15 patients and 18 healthy subjects. The $\chi^{2}$ test showed that the difference in prevalence of streptococcal strains between periodontitis patients and healthy controls was significant only for $S$. sanguinis, which was found significantly more often in healthy subjects $(P=0.001)$. Significant differences between males and females and between smokers and non-smokers with respect to prevalence of oral streptococci were not found.
The relationship with age and periodontal indices of streptococcal strains was analysed using a Mann-Whitney test. Table 3 presents the $P$ values for the significant association between the absence of $S$. sanguinis and high values of API, gingival index, BOP, CAL and PD.

This study analysed the prevalence of oral streptococci in patients suffering from aggressive periodontitis and in healthy control individuals. Special emphasis was laid on the correct species identification of the streptococci. As it is known that conventional biochemical tests may fail to discern certain species of viridans streptococci, the recently established MALDI-TOF-MS methodology (Smole et al., 2002; Rupf et al., 2005; Friedrichs et al., 2007) was used and adjusted for this study.

The results indicated that, among the 11 species cultured from patients and healthy individuals, only one species $-S$. sanguinis - showed significant differences in colonization rates for the two groups, and was associated with health rather than disease.

It has been demonstrated previously that $S$. sanguinis is a beneficial bacterium in the prevention of dental caries. The antagonism with $S$. mutans has been known for many years. Early colonization with $S$. sanguinis is significantly correlated with a delay in colonization by $S$. mutans. After $S$. mutans colonization, the levels of $S$. sanguinis decrease (Caufield et al., 2000; Kreth et al., 2005).

Several other studies have focused on the in vitro relationship between $S$. sanguinis and some of the periodontal pathogens. Hillman et al. (1985) showed that, in samples from oral sites where S. sanguinis was detected, Tannerella forsythia was present in $1 \%$ of the cases, and in samples where S. sanguinis was not detected, Tannerella forsythia was present in $10 \%$ of the oral sites. They also showed that one-third of $S$. sanguinis strains tested were able to inhibit Prevotella intermedia BS6. In vitro growth of Aggregatibacter actinomycetemcomitans is inhibited by the

Table 2. Distribution of oral streptococcal species in patients and healthy subjects

$n$, Number of patients/healthy subjects colonized with oral streptococci.

\begin{tabular}{|c|c|c|c|c|c|}
\hline \multirow[t]{2}{*}{ Oral streptococci } & \multicolumn{2}{|c|}{ Colonized periodontitis patients } & \multicolumn{2}{|c|}{ Colonized healthy subjects } & \multirow[t]{2}{*}{$P$ value $\left(\chi^{2}\right.$ test $)$} \\
\hline & $n$ & $\%$ & $n$ & $\%$ & \\
\hline S. mitis & 12 & 36.36 & 8 & 40.00 & 0.723 \\
\hline S. sanguinis & 15 & 45.45 & 18 & 90.00 & 0.001 \\
\hline S. oralis & 24 & 72.72 & 14 & 70.00 & 0.723 \\
\hline S. gordonii & 8 & 24.24 & 0 & 0.00 & 0.017 \\
\hline S. cristatus & 1 & 3.03 & 1 & 5.00 & 0.715 \\
\hline S. sinensis & 1 & 3.03 & 0 & 0.00 & 0.432 \\
\hline S. anginosus & 3 & 9.09 & 1 & 5.00 & 0.585 \\
\hline S. salivarius & 1 & 3.03 & 0 & 0.00 & 0.432 \\
\hline S. constellatus & 7 & 21.21 & 1 & 5.00 & 0.110 \\
\hline S. parasanguinis & 1 & 3.03 & 1 & 5.00 & 0.715 \\
\hline S. mutans & 0 & 0.00 & 1 & 5.00 & 0.195 \\
\hline
\end{tabular}


Table 3. Association between absence of $S$. sanguinis and high values of periodontal indices

\begin{tabular}{|lc|}
\hline & $P$ value \\
\hline Age & 0.446 \\
OHI & 0.010 \\
Lobene & 0.002 \\
API & $<0.001$ \\
BOP & 0.004 \\
$\mathrm{CAL}$ (total) & 0.007 \\
$\mathrm{CAL}^{\dagger}$ (sites) & 0.002 \\
$\mathrm{PD}^{*}$ (total) & 0.001 \\
$\mathrm{PD}^{\dagger}$ (sites) & 0.002 \\
\hline
\end{tabular}

${ }^{\star}$ Mean of all teeth.

¥Mean of the four sampling sites.

hydrogen peroxide produced by S. sanguinis (Hillman et al., 1985; Hillman \& Socransky, 1982). However, A. actinomycetemcomitans may produce a bacteriocin that can kill S. sanguinis (Hammond et al., 1984), so there is an inverse relationship between these bacteria. In a complex ecosystem such as dental biofilm, these relationships may contribute to the transition from health to disease. Also, Teughels et al. (2007) showed in an in vitro study that $S$. sanguinis (as well as $S$. mitis and Streptococcus salivarius) has protective properties that interfere with A. actinomycetemcomitans colonization of epithelial cells. All of these data suggest that $S$. sanguinis may serve as a 'protective' bacterium as defined by Quirynen et al. (2001). The effect of $S$. sanguinis on the presence of Porphyromonas gingivalis is still under debate. Whilst Hillman et al. (1985) showed that $S$. sanguinis had a minimal effect on the presence of Porphyromonas gingivalis, another study from China showed that pre-colonization and superinfection with $S$. sanguinis reduced the level of Porphyromonas gingivalis in experimental rats (Zhang et al., 2000).

In clinical studies, Haffajee et al. (1998) showed that $S$. sanguinis and $S$. oralis did not differ among the subject group of periodontitis patients, healthy controls and elders with a well-maintained periodontitis, and Dowsett et al. (2002) reported that $S$. sanguinis was more prevalent in shallow sites but was not the species associated with health. These discrepancies could be due to different subject inclusion and exclusion criteria and work protocols. These latter two clinical studies based their identification on a chequerboard DNA-DNA hybridization assay, whilst previously mentioned in vitro studies used cultivation. Our study combined cultivation with MALDI-TOF-MS.

A recent study (Maestre et al., 2007) showed that of all of the bacteria from subgingival plaques (including anaerobic bacteria), $S$. oralis and $S$. mitis were the most prevalent species, with a frequency of detection of approximately $70 \%$, whilst S. sanguinis was isolated in only $23 \%$ of cases. The three species were the only oral streptococci identified in this study, and 33/125 strains could not be identified to the species level. This is in keeping with the majority of earlier studies which focused on two or three species of oral streptococci only. The reason could be that only conventional biochemical methods were used for identification. With our study, we were able to employ MALDI-TOF-MS methodology, which allowed us to identify a larger number of different species. In our study, S. oralis was also the most prevalent oral streptococcus in both groups of subjects (present in $70 \%$ of cases), but S. sanguinis was the second most common isolate (present in $90 \%$ of healthy subjects and $45 \%$ of periodontitis patients). S. mitis was present in both groups in 36-40\% of cases. Interestingly, all eight strains of Streptococcus gordonii were isolated from periodontitis patients, but the difference between the two groups was not significant $(P>0.003)$. Lamont et al. (2002) and Daep et al. (2006) demonstrated that S. gordonii plays a role in colonization with Porphyromonas gingivalis, whilst Haffajee et al. (2006) found this species to be more prevalent in healthy subgingival sites. Further studies are needed to clarify the possible relationship between $S$. gordonii and periodontal status.

In conclusion, aggressive periodontitis seems to be associated with a loss of colonization with $S$. sanguinis. Whether or not $S$. sanguinis offers protection against aggressive periodontitis needs to be determined. Otherwise, there were no significant differences in the distribution of oral streptococcal species between patients and healthy subjects.

\section{ACKNOWLEDGEMENTS}

The authors thank Annett Hennig-Rolle and Helga Stache for technical assistance and Oana Brosteanu for statistical analysis.

\section{REFERENCES}

Caufield, P. W., Dasanayake, A. P., Li, Y., Pan, Y., Hsu, Y. \& Hardin, M. (2000). Natural history of Streptococcus sanguinis in the oral cavity of infants: evidence for a discrete window of infectivity. Infect Immun 68, 4018-4023.

Daep, C. A., James, D. M., Lamont, R. J. \& Demuth, D. R. (2006). Structural characterization of peptide-mediated inhibition of Porphyromonas gingivalis biofilm formation. Infect Immun 74, 5756-5762.

Dowsett, S. A., Kowolik, M. J., Archila, L. A., Eckert, G. J. \& LeBlanc, D. J. (2002). Subgingival microbiota of indigenous Indians of Central America. J Clin Periodontol 29, 159-167.

Frandsen, E. V., Pedrezzoli, V. \& Killian, M. (1991). Ecology of viridans streptococci in the oral cavity and pharynx. Oral Microbiol Immunol 6, 129-133.

Friedrichs, C., Rodloff, A. C., Chhatwal, S., Schellenberger, W. \& Eschrich, K. (2007). Rapid identification of viridans streptococci by mass spectrometric discrimination. J Clin Microbiol 45, 2392-2397.

Haffajee, A. D., Cugini, M. A., Tanner, A., Pollack, R. P., Smith, C., Kent, R. L. \& Socransky, S. S. (1998). Subgingival microbiota in healthy, well-maintained elder and periodontitis subjects. J Clin Periodontol 25, 346-353.

Haffajee, A. D., Teles, R. P. \& Socransky, S. S. (2006). Association of Eubacterium nodatum and Treponema denticola with human periodontitis lesions. Oral Microbiol Immunol 21, 269-282. 
Hammond, B. F., Stevens, R. H., Bonner, P. \& Lillard, S. E. (1984). Toxicity of Actinobacillus actinomycetemcomitans extracts for crevicular bacteria. J Dent Res 63, 263 (abstract)

Hillman, J. D. \& Socransky, S. S. (1982). Bacteria interference in the oral ecology of Actinobacillus actinomycetemcomitans and its relationship to human periodontosis. Arch Oral Biol 27, 75-77.

Hillman, J. D., Socransky, S. S. \& Shivers, M. (1985). The relationships between streptococcal species and periodontopathic bacteria in human dental plaque. Arch Oral Biol 30, 791-795.

Hintao, J., Teanpaisan, R., Chonqsuvivatwong, V., Ratarasan, C. \& Dahlen, G. (2007). The microbiological profiles of saliva, supragingival and subgingival plaque and dental caries in adults with and without type 2 diabetes mellitus. Oral Microbiol Immunol 22, 175-181.

Kreth, J., Merritt, J., Shi, W. \& Qi, F. (2005). Competition and coexistence between Streptococcus mutans and Streptococcus sanguinis in the dental biofilm. J Bacteriol 187, 7193-7203.

Lamont, R. J., EI-Sabaeny, A., Park, Y., Cook, G. S., Costerton, J. W. \& Demuth, D. R. (2002). Role of the Streptococcus gordonii SspB protein in the development of Porphyromonas gingivalis biofilms on streptococcal substrates. Microbiology 148, 1627-1636.

Maestre, J. R., Bascones, A., Sánchez, P., Matesanz, P., Aguilar, L., Giménez, M. J., Pérez-Balcabao, I., Granizo, J. J. \& Prieto, J. (2007). Odontogenic bacteria in periodontal disease and resistance patterns to common antibiotics used as treatment and prophylaxis in odontology in Spain. Rev Esp Quimioter 20, 61-67.

Marsh, P. D. (1991). Sugar, fluoride, $\mathrm{pH}$ and microbial homeostasis in dental plaque. Proc Finn Dent Soc 87, 515-525.

Marsh, P. D. (2004). Dental plaque as a microbial biofilm. Caries Res 38, 204-211.

Marsh, P. D. \& Bradshaw, D. J. (1999). Microbial community aspects of dental plaque. In Dental Plaque Revisited: Oral Biofilms in Health and Disease, pp. 237-253. Edited by H. N. Newman \& M. Wilson. Cardiff: BioLine Publications.
Quirynen, M., DeSoete, M., Dierickx, K. \& van Steenberghe, D. (2001). The intra-oral translocation of periodontal pathogens jeopardises the outcome of periodontal therapy. A review of the literature. J Clin Periodontol 28, 499-507.

Rupf, S., Breitung, K., Schellenberger, W., Merte, K., Kneist, S. \& Eschrich, K. (2005). Differentiation of mutans streptococci by intact cell matrix-assisted laser desorption/ionization time-of-flight mass spectrometry. Oral Microbiol Immunol 20, 267-273.

Smole, S. C., King, L. A., Leopold, P. E. \& Arbeit, R. D. (2002). Sample preparation of Gram positive bacteria for identification by matrix assisted laser desorption/ionization time-of-flight. $J$ Microbiol Methods 48, 107-115.

Socransky, S. S., Haffajee, A. D. \& Cugini, M. A. (1998). Microbial complexes in subgingival plaque. J Clin Periodontol 25, 134-144.

Teughels, W., Haake, K. S., Sliepen, I., Pauwels, M., van Eldere, J., Cassiman, J. J. \& Quirynen, M. (2007). Bacteria interfere with $A$. actinomycetemcomitans colonization. J Dent Res 86, 611-617.

Theilade, E., Wright, W., Borglum Jensen, S. \& Loe, H. (1966). Experimental gingivitis in man. II. A longitudinal clinical and microbiological investigation. J Periodont Res 1, 1-13.

Van der Reijden, W. A., Dellemijn-Kippuw, N., Stijne-van Ness, A. M., de Soet, J. J. \& van Winkelhoff, A. J. (2001). Mutans streptococci in subgingival plaque of treated and untreated patients with periodontitis. J Clin Periodontol 28, 686-691.

Vapnik, V. N. (1995). The Nature of Statistical Learning Theory. Berlin: Springer-Verlag.

Wiebe, C. B. \& Putnins, E. E. (2000). The periodontal disease classification system of the American Academy of Periodontology an update. J Can Dent Assoc 66, 594-597.

Zhang, J. C., Zhou, C., Wu, B. \& Zhang, Y. H. (2000). Investigation on interaction between Streptococcus sanguis and Porphyromonas gingivalis in specific pathogen-free rats. Chin J Dent Res 3, 5-9. 